\title{
Why lawyers should deal with Nudges
}

Niels Petersen

2015-04-18T08:40:11

Emanuel Towfigh and Christian Traxler have asked why the nudging debate has arrived so late in the German legal discourse. They argue that this is due to a mixture of reasons related to legal culture and legal education. I agree with their analysis. So let me address one question that both authors do not touch. Why should lawyers deal with the question of nudging? Wouldn't this rather be a task for psychologists or behavioral economists? Prima facie, there seems to be a lot in favor of leaving the discussion on nudges to social scientists. A nudge seeks to alter people's behavior without restraining choices. In order to influence people's behavior, however, you have to analyze behavioral patterns, which is impossible without empirical methods.

Nevertheless, there are two reasons why lawyers should not totally leave the field to social scientists. First, the claim that the legislature should use nudges or soft paternalism to influence individual behavior is a normative one. Lawyers are better suited than empirically minded social scientists to balance the positive effects of such a policy against competing normative aims. However, the more important point is probably a second one. Social scientists usually don't deal with overarching policy questions. It is at the same time the strength and the weakness of orthodox empirical social sciences that they are based on a rather rigorous methodology. These methodological constraints also influence the research questions. Social scientists often prefer small " $\mathrm{t}$ " theories to holistic ones because their methods are only applicable to strictly limited questions. For this reason, they usually shy away from important policy questions because their methodology does not allow them to answer these questions.

In this respect, lawyers have a comparative advantage. They can often base normative conclusions on educated guesses or assumptions without violating the conventions of their discipline - something that would be unthinkable in economics or psychology. Consequently, even if the nudging debate has arrived late in German legal scholarship, it is important that it has arrived. If the legal community did not deal with the topic, soft paternalism would probably be an issue left to political pragmatism without deeper scientific reflection.

(cc) BY-NC-ND 